rev.relac.int.estrateg.segur.9(1):179-197,2014

\title{
PREPARAR EL POST-CONFLICTO EN COLOMBIA DESDE LOS PROGRAMAS DE DESARROLLO Y PAZ: RETOS Y LECCIONES APRENDIDAS PARA LA COOPERACIÓN INTERNACIONAL Y LAS EMPRESAS*
}

\author{
Miguel Barreto Henriques**
}

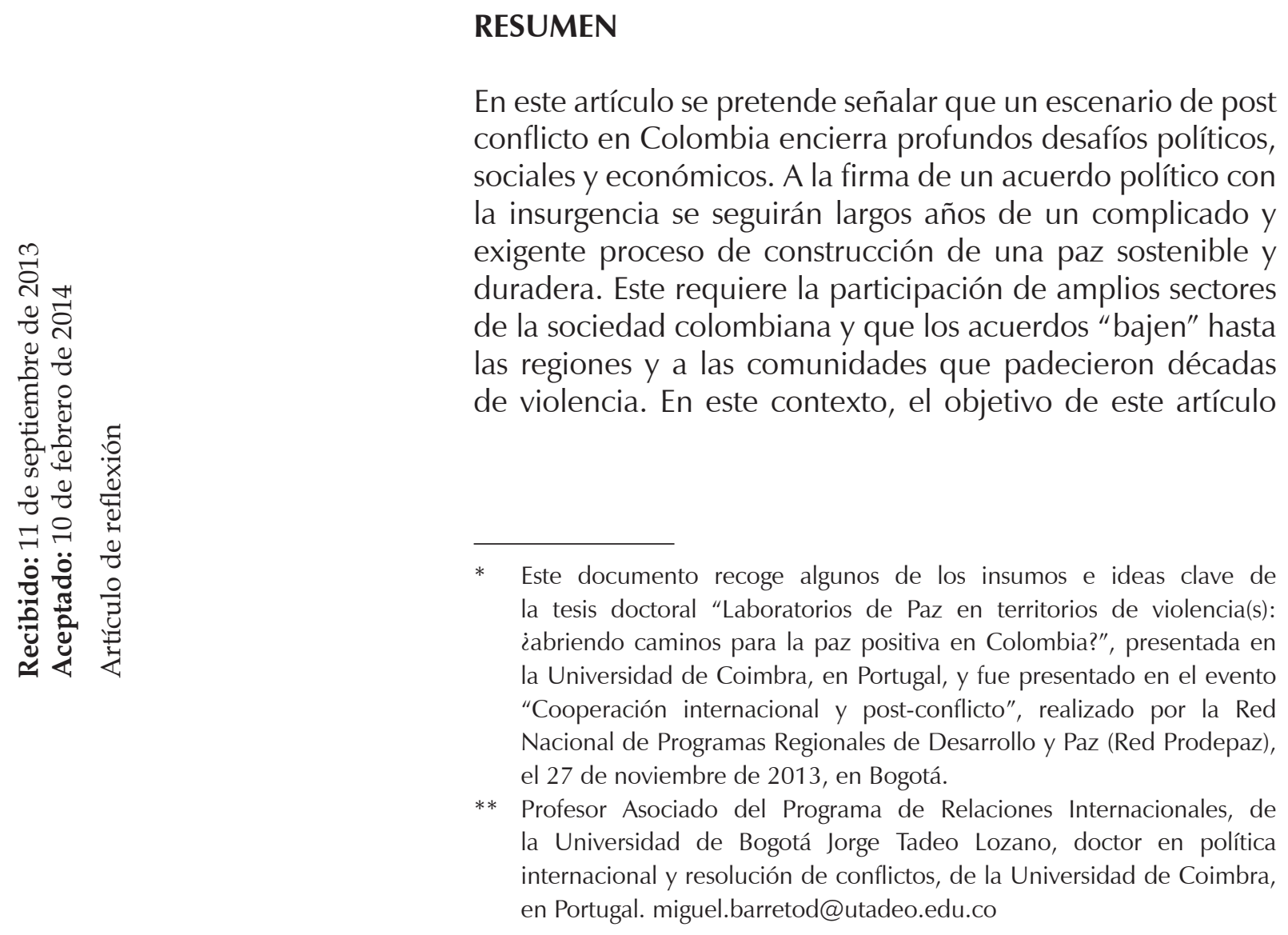


es, en primer lugar, subrayar el potencial de los Programas de Desarrollo y Paz (PDP) en un escenario de post conflicto en Colombia, en cuanto modelos consolidados de construcción de paz desde la sociedad civil y las regiones; y, en segundo lugar, analizar cómo la cooperación internacional y las empresas podrán respaldar estas iniciativas en este nuevo entorno político nacional, teniendo en cuenta lecciones aprendidas de la experiencia de colaboración y alianza con los PDP.

Palabras clave: Cooperación al desarrollo, Programas de Desarrollo y Paz (PDP), construcción de paz, post conflicto

\title{
PREPARING FOR POST-CONFLICT IN COLOMBIA FROM DEVELOPMENT AND PEACE PROGRAMS: CHALLENGES AND LEARNED LESSONS FOR INTERNATIONAL COOPERATION AND COMPANIES
}

\begin{abstract}
This article aims to underline that a potential post-conflict scenario in Colombia holds profound political, social and economic challenges. After the signing of a political agreement with the guerrilla, the country will face a complicated and demanding process of peace-building aiming to generate a sustainable and everlasting peace. This process requires the participation of broad sectors of Colombian society and that the agreements are implemented in the regions and communities who experienced decades of violence. In this context, the objective if this paper is, firstly, to highlight the potential of the Peace and Development Programs (PDP), as consolidated regional models of peace-building put in practice by civil society; and, secondly, to analyze how international aid and enterprises could support these initiatives in a new national political context, taking into account lessons learnt from the previous experience of alliance and collaboration with the PDP.
\end{abstract}

Keywords: Development aid, Development and Peace program (PDP), peace-building, postconflict

\section{PREPARE PÓS-CONFLITO NA COLÔMBIA, DE PROGRAMAS DE DESENVOLVIMENTO E PAZ: DESAFIOS E LIÇÕES APRENDIDAS PARA A COOPERAÇÃO INTERNACIONAL E NEGÓCIOS}

\section{RESUMO}

Este artigo pretende assinalar que um cenário de pós-conflito na Colômbia implica profundos desafios políticos, sociais e econômicos. Para a assinatura de um acordo político com a insurgência serão necessários longos anos de um complicado e exigente processo de construção 
de uma paz sustentável e duradoura. Isso requer a participação de amplos sectores da sociedade colombiana e que os acordos "baixem" às regiões e às comunidades que padeceram por décadas a violência. Neste contexto, o objetivo deste artigo é, em primeiro lugar, enfatizar o potencial dos Programas de Desenvolvimento e Paz PDP em uns cenários de pós-conflito na Colômbia, como modelos consolidados de construção de paz desde a sociedade civil e as regiões; e, em segundo lugar, analisar como a cooperação internacional e as empresas poderão apoiar estas iniciativas neste novo contexto político nacional, levando em consideração as lições aprendidas da experiência de colaboração e aliança com os PDP.

Palavras chave: Cooperação para o desenvolvimento, Programas de Desenvolvimento e Paz PDP, construção de paz, pós-conflito

\section{INTRODUCCIÓN}

Colombia se enfrenta actualmente a uno de los mayores desafíos de su historia reciente: poner definitivamente fin a un sangriento conflicto armado de cinco décadas y construir un país y una sociedad en paz.

La posibilidad de un acuerdo político entre las FARC y el gobierno nacional en la Habana permite soñar con ese escenario, pero también genera varios interrogantes: ¿Cómo será la etapa del post conflicto en Colombia? ¿Qué desafíos se presentarán al país y a la sociedad? ¿Qué riesgos y peligros podrán enfrentar? ¿Cómo se pacificará política, social y culturalmente la sociedad y las regiones? ¿Cómo se procesará la construcción de una paz duradera y sostenible a largo plazo? ¿Qué actores podrán desempeñar un papel importante en este cuadro?

El presente documento subrayará la importancia de la sociedad civil en la construcción de una paz sostenible y duradera, enfocándose en el rol que podrán jugar los Programas de Desarrollo y Paz (PDP), la cooperación internacional y las empresas en un escenario de post conflicto en Colombia. Buscará, en primer lugar, analizar y enfatizar el potencial de los PDP como actores y modelos de construcción de paz desde las regiones; $y$, en segundo lugar, evaluar en qué medida y cómo la cooperación internacional y las empresas privadas podrán respaldar estos procesos en una etapa de post conflicto, teniendo en cuenta lecciones aprendidas de la previa experiencia de colaboración y alianza con los PDP.

\section{LOS RETOS DEL POST CONFLICTO EN COLOMBIA}

Si algo nos ha mostrado las experiencias de varios países en el mundo, como El Salvador, Guatemala o Irlanda del Norte, es que un escenario de post conflicto trae tantos desafíos políticos y sociales a un país como el mismo proceso de paz y el conflicto armado. 
Un proceso de negociación y un acuerdo de paz tienen un alcance limitado. Permiten un primer paso fundamental e indispensable para la paz: el silenciamiento de los fusiles de los actores armados, pero no necesariamente eliminar los gérmenes de violencia y las raíces de la conflictividad. Inciden tan solo sobre una de las dimensiones de un conflicto -los aparatos armados de los grupos ilegales y sus comandos políticos (Granada, Restrepo y Vargas, 2009: 103). Por lo tanto, no son garantía de un país verdaderamente pacificado, sino de una paz frágil, limitada e imperfecta. No generan una paz sostenible y duradera, que sea transversal a todos los niveles y esferas de relaciones sociales.

Colombia es un país cuya violencia es un fenómeno particularmente complejo y muldimensional. Adopta diversas modalidades, formas y expresiones, en las cuales se interconectan y se cruzan violencia insurgente, paramilitar, mafiosa, criminal y narcotraficante, y no se circunscriben a la existencia de grupos alzados en armas (McDonald, 1997: 1). La violencia en Colombia está enraizada y es alimentada y sostenida por factores políticos y socio-económicos estructurales.

El conflicto armado está enmarcado en procesos históricos de largo plazo y de varias índoles, que se tradujeron en un panorama de marginalización política, social y económica de amplios sectores de la población colombiana, en particular, del campesinado, y en la exclusión, o precaria integración, de algunas regiones y zonas de Colombia, del desarrollo, la democracia y las instituciones del Estado. Se cristalizó una brecha entre el centro y periferia del país, entre "dos Colombias", asimétricas política, social y económicamente, factor que ha alimentado históricamente el desarrollo de grupos armados ilegales y la emergencia de violencia bajo distintas modalidades y formas. La violencia armada ha emergido sociológicamente y se ha circunscrito sobre todo a la Colombia rural.

Por lo tanto, si los esfuerzos de pacificación de Colombia se limitan a la dimensión de meros acuerdos entre el Estado y la insurgencia, y no se abordan las causas de la conflictividad, hay un gran riesgo que el país no sea verdaderamente pacificado y que la violencia reincida o se transfigure y asuma nuevas formas y modalidades en los territorios periféricos y rurales del país, y se sigan generando y alimentado grupos con la capacidad de desafiar el monopolio legítimo de la fuerza sea en la forma de guerrillas, de grupos paramilitares, narcotraficantes o pandillas. De hecho, el riesgo de no abordar las causas subyacentes de los conflictos es tan grande como no tratar los síntomas de una enfermedad (Burton, 1990).

El fenómeno de las "nuevas bandas emergentes" o BACRIM, que se sucedieron al proceso formal de desmovilización de las AUC, o de las "maras" en Centroamérica, en donde una "guerra de frentes se convirtió en una guerra de todas las esquinas" (Vincenti, 2008), revela, en gran medida, esta realidad y constituye un alerta para los peligros y riesgos de un escenario post-negociación.

Por esta razón, es importante subrayar que la paz no se materializará en Colombia, como un "café instantáneo", en el día que se firme un acuerdo entre el gobierno y las FARC. La 
construcción de una paz duradera, sostenible y positiva ${ }^{1}$ implica un proceso mucho más largo y que requiere la participación de muchos más actores de la sociedad, más allá del Estado y de los actores armados.

Pasará por las distintas esferas y niveles de la organización social, por las políticas macro definidas desde la Casa de Nariño, en Bogotá, pero también por cada región, territorio y vereda del país, que tendrán que generar nuevas formas de convivencia. Implica un "desarme" social, político, económico y cultural de la sociedad colombiana, y el cambio de una cultura de violencia por una cultura de paz. Es un proceso paulatino y progresivo que se juega a varias escalas y niveles e implica la participación amplia de la población. Usando una metáfora de Lederach (2007: 37), uno de los más reputados teóricos en temas de resolución de conflictos, para construir la "casa de la paz", se requiere un trabajo tanto en el techo como en los cimientos, tanto en la cima, como la base de la pirámide del conflicto; todos los niveles son igualmente importantes para que esta se sostenga y no caiga.

En este sentido, todos los actores políticos, económicos y sociales del país, desde los partidos, a las universidades, de los empresarios, a las ONG, de las gobernaciones a las comunidades de base, de los individuos a las organizaciones de la comunidad internacional, tendrán un rol a desempeñar en el post conflicto y un aporte a dar en la construcción de una Colombia en paz.

\section{2. ¿ ¿QUÉ ROL PODRÁ DESEMPEÑAR LA SOCIEDAD CIVIL EN UN ESCENARIO DE POST CONFLICTO EN COLOMBIA? ANÁLISIS DE LOS CASOS DE LOS PDP, DE LA COOPERACIÓN INTERNACIONAL Y DE LAS EMPRESAS}

En la medida en que un proceso de construcción de paz es mucho más amplio que un proceso de paz, se figura como fundamental la participación de la sociedad civil en un escenario de post conflicto.

Su importancia resulta de la necesidad de garantizar la sostenibilidad de la paz. Un proceso de paz solo es sostenible si es apropiado por la población (Mouly, 2011: 304). La construcción de la paz debe partir y enraizarse en el mismo suelo en que el conflicto se desarrolló (Lederach,

1. El concepto de "paz positiva", cuñado por Johan Galtung (1996), amplió el significado convencional de la paz, simplemente de la antítesis de la guerra y revaluó el concepto de violencia de su sentido y connotación tradicional -violencia física- para darle una significación más amplia. Desde este punto de vista, la paz no es solamente la ausencia de la guerra, estos no son elementos equivalentes. Lo que le anima es una visión de la paz, no como antónimo de la guerra, sino de violencias. Galtung concibe una paz definida positivamente, que implica una reestructuración profunda de las relaciones humanas. Transmite un concepto amplio y denso de paz, uno que implica mucho más que el silenciamiento de los fusiles. Se basa en una visión integral de la paz y los conflictos, que establece un vínculo claro y fuerte entre el desarrollo, la justicia social y los temas de la paz. 
1997: 107) y solo se logrará mediante la participación de los protagonistas y las víctimas de la violencia en cada región y localidad del conflicto (McDonald, 1998: 93). Para que la paz se consolide y gane raíces hay que crear la paz entre vecinos y en el seno de las comunidades. La "gente" es la clave para desarrollar una infraestructura y una cultura de paz a largo plazo.

En las últimas dos décadas han emergido en Colombia un alto número de iniciativas de construcción de paz desde la sociedad civil, que han demostrado otro lado y dimensión del conflicto y han configurado este país como un escenario simultáneamente de guerra y de paz (o paces).

\subsection{LOS PDP EN UN ESCENARIO DE POST-CONFLICTO}

En este cuadro, los Programas de Desarrollo y Paz se han destacado como una de las más interesantes, ambiciosas y originales iniciativas de construcción de paz puestas en marcha desde la sociedad civil en Colombia.

Basados en la filosofía y metodología originales del Programa de Desarrollo y Paz del Magdalena Medio (PDPMM), su enfoque para la paz se ha expandido a diversas regiones del país, con resultados notables en el territorio, en términos de apertura de caminos alternativos para la paz, a partir de procesos de desarrollo y de integración de grupos sociales excluidos a nivel local y regional.

Los PDP representan hoy en Colombia un modelo consolidado de construcción de paz positiva y sostenible desde la base, puesto en marcha a partir de la especificidad de las regiones y de los territorios marginados del país, por organizaciones de la sociedad civil, en articulación con las instituciones del Estado y con la cooperación internacional (Barreto Henriques, 2012).

En este cuadro se impone la cuestión: ¿Qué rol podrían cumplir los PDP en un escenario de post-conflicto?

Un acuerdo político con la guerrilla conferiría necesariamente a los PDP un nuevo rol y misión. Sus procesos sociales se han desarrollado, hasta el momento, en medio del conflicto armado, en territorios de gran precariedad y violencia y contextos supremamente difíciles. No obstante, han obtenido impactos positivos a nivel local en términos de resistencia civil, generación de una cultura de paz y de ciudadanía, inclusión socioeconómica de campesinos y trabajo con las comunidades de base.

En esta medida, un nuevo contexto político y social de paz potenciaría el rol de los PDP en Colombia e incrementaría sus posibilidades de trabajo de construcción de paz con las comunidades a nivel territorial.

Su potencial para un escenario de post conflicto reside en varios factores y elementos que se han desarrollado e implementado en casi 20 años de labor para la paz: 
En primer lugar, los PDP tienen en cuenta la especificidad del conflicto armado y de la violencia en Colombia. Configuran modelos de intervención territorial, para las zonas periféricas del país, los espacios geográficos y sociales que han alimentado históricamente el conflicto.

Muestran líneas de acción concretas para la construcción de la paz duradera, en estos territorios marginados del país, teniendo en cuenta los factores estructuradores del conflicto en Colombia, estableciendo una metodología válida de aproximación a las causas profundas del conflicto y las estructuras de exclusión que impiden el ejercicio de la ciudadanía para una buena parte de la población.

En segundo lugar, han abierto una vía regional para la paz en el país, a partir de procesos sociales a nivel micro, que han descentralizado la construcción de paz en Colombia (Barreto Henriques, 2009: 559).

Estos elementos son fundamentales para estructurar un proceso integral de construcción de paz en el post conflicto, pues una política o un proceso de paz en Colombia que no tenga en cuenta la variable territorial y las particularidades regionales está condenada a fracasar o a obtener impactos mitigados. Para que sea sostenible y duradera no puede basarse en un enfoque estrictamente nacional. Necesita que encierre escalas y enfoques espaciales distintos y agendas locales y regionales de paz, pasando necesariamente por el involucramiento de las regiones, la participación activa de las comunidades y de la sociedad civil organizada a partir de los territorios, de forma que pueda desactivar expresiones violentas, y enraizar dinámicas de resolución pacífica de conflictos, de cultura de paz y de inclusión política, social, económica, y regional.

En tercer lugar, el potencial de construcción de paz de los PDP para un marco de post-conflicto reside en la integralidad de sus elementos. Se destacan por ser un programa multidimensional, que integra simultáneamente diversas áreas y componentes de la construcción de la paz, desde la vertiente económica, a la cultura de paz y al fortalecimiento institucional. Es una iniciativa de aproximación integral y holística para la paz, que tiene como horizonte la construcción de la paz positiva y no solo la ausencia de la guerra, elemento central en un escenario de post conflicto.

En cuarto lugar, su aporte radica especialmente en su propuesta de conjugar la paz con el tema del desarrollo. Construir la paz mediante procesos de inclusión económica y de desarrollo humano sostenible, aparece de forma innovadora e indisociable con estos programas. Los PDP fueron pioneros en Colombia en su intento de combinar y "casar" la construcción de paz desde la base con procesos de desarrollo, lo que ha permitido una cierta sostenibilidad de los procesos sociales, un arraigo al territorio, y encierra un potencial de inmunización de sectores sociales potencialmente en riesgo, como los campesinos y los jóvenes.

Parten del principio de que la construcción de la paz pasa por generar nuevas condiciones de vida, la inclusión para la gente en el campo y por el desarrollo socioeconómico de las 
comunidades (Saavedra y Ojeda, 2006: 32), lo que se concibe como esencial, en la medida en que la falta de horizontes de vida de la gente en el campo es un factor determinante que alimenta el conflicto armado, y empuja a millares de familias hacia la economía de la coca. En esta medida, intervenir sobre esta raíz del conflicto es un canal privilegiado para su transformación.

En este contexto, han logrado construir o preservar espacios de economía lícita, confiriendo alternativas de ocupación económica y dando nuevas posibilidades de vida a varios sectores sociales como los jóvenes de las comunas de Barrancabermeja o los campesinos del sur del Bolívar y del Patía, incidiendo de alguna forma sobre la violencia estructural y directa que se hace sentir sobre la población más excluida de la región. Mediante estos procesos se han robado hombres a la guerra, pero también espacio político y social.

De esta forma, los PDP se figuran como instrumentos de promoción de desarrollo rural integral y micro-plataformas de inclusión de los campesinos, en la vida social, económica, política y productiva, sector social que seguirá en una posición delicada y de riesgo en un escenario de post conflicto.

En quinto lugar, los PDP desarrollan mecanismos y procesos que permiten "democratizar la democracia" (Sousa Santos, 2003) a nivel regional y local y fortalecer las instituciones. Han puesto en marcha diversos procesos de fomento de una democracia participativa a nivel local y regional, con miras a acercar los ciudadanos a las instituciones, y fomentar un nuevo modelo de relación entre gobernantes y gobernados, mediante procesos de articulación entre el sector público y el privado, y entre la sociedad civil y las alcaldías y gobiernos departamentales, a través de programas de rendición pública de cuentas y presupuestos participativos, y mecanismos participativos de planificación como los "Planes de Vida" en el Cauca y Nariño y los "Núcleos de Pobladores" en el Magdalena Medio.

En sexto lugar, los PDP permiten generar bolsas de una cultura de la paz, es decir, micro espacios y expresiones de paz, en los cuales los valores de la civilidad y la solidaridad se han sobrepuesto a las dinámicas de la violencia. Desarrollan un trabajo valiosísimo en el sentido de desarrollar y generar una nueva cultura en los territorios y veredas de las regiones y cambiar los paradigmas de pensamiento de las comunidades. Fomentan la exploración y desarrollo desde lo cotidiano de nuevas formas de relación e interrelación que superan la polarización del conflicto armado, deslegitiman cultural y socialmente la guerra y el recurso a la violencia. Aquí la paz emerge como un proceso que se va construyendo cotidianamente desde las veredas de las regiones, corporizando una "paz del día a día" (Richmond, 2008: 109). Estos procesos paulatinos son los que permiten centar los cimientos de una paz sostenible y duradera.

Por último, el enfoque de los PDP permite de cierta forma reconciliar las "Dos Colombias", o sea, integrar los territorios y sectores sociales históricamente marginados, de las instituciones, la 
democracia y el desarrollo, aproximando las veredas de las regiones y la totalidad del territorio nacional a la institucionalidad, al Estado de Derecho, y al desarrollo; llevando la democracia y una cultura democrática y ciudadana a las veredas más remotas, proveyendo servicios públicos; ayudando a llenar el profundo vacío institucional; repartiendo el bienestar y los dividendos del desarrollo regional entre todos sin excepciones; disminuyendo el foso centro-periferia; ayudando a superar las fronteras y periferias geográficas, políticas, sociales y económicas. En cierta medida, es una forma de construcción del Estado y de la Nación, proceso que es fundamental para la construcción de una Colombia en paz.

\subsection{LA COOPERACIÓN INTERNACIONAL Y LAS EMPRESAS EN UN ESCENARIO DE POST CONFLICTO}

Se ha enfatizado en este documento que un proceso de construcción de paz y de reconstrucción post-bélica requiere la participación de amplios sectores de la sociedad.

En seguida, nos referiremos a dos actores que podrán desempeñar un rol importante en una etapa de post conflicto en Colombia como aliados de los PDP -la cooperación internacional y las empresas. Estos son actores esenciales para la consolidación de una economía de paz, que sustente la construcción de una paz duradera. Como nos han demostrado los PDP en sus procesos sociales a nivel comunitario, el desarrollo es un medio para la paz. Así, estos dos actores, como protagonistas fundamentales del desarrollo, pueden hacer un aporte relevante en esto.

Su importancia en este marco se ve agregada por el papel esencial que han desempeñado la cooperación internacional y las empresas, en el pasado y presente, como aliados de los PDP y protagonistas de sus procesos y vida cotidiana. Han garantizado su viabilidad financiera y su buen financiamiento. La cooperación europea respaldó política y financieramente durante años, por intermedio de los Laboratorios de Paz, estas iniciativas, permitiendo que se fortalecieran, se consolidaran y se expandieran.

En breve, se podrán encontrar frente a un nuevo desafío: respaldar política y financieramente una nueva fase de los PDP en un escenario distinto, pero igualmente exigente para la construcción de paz: el post conflicto. Si los PDP vendrán a jugar una función importante en un contexto de post conflicto, necesariamente dependerán de la cooperación internacional y de las empresas, sus naturales e históricos aliados, para darles sostenibilidad y permitir que sigan su rol. Una nueva fase de los Laboratorios de Paz podría abrirse para la cooperación europea o podrían generarse nuevos mecanismos de apoyo a los PDP a lo ancho del territorio nacional colombiano.

Pero no solo la cooperación internacional y europea tienen un papel potencial a desempeñar en este contexto, empresas como Ecopetrol, ISA e ISAGEN han estado, desde el inicio, vinculadas 
a los PDP, especialmente en zonas como el Magdalena Medio y el Oriente Antioqueño, siendo partícipes fundamentales de sus procesos.

En esta medida, su rol podría extenderse en un escenario de post conflicto, especialmente en un contexto en el cual los fondos de la cooperación internacional, por la crisis de la UE y de los EEUU, y por el crecimiento económico notable de Colombia, configuran una tendencia para el "cierre progresivo del grifo" de la ayuda al desarrollo en un país de renta media. La inversión de las empresas en la construcción de la paz en Colombia, y, particularmente, en los PDP, es una cuestión de responsabilidad social, pero también de generar una economía de paz, que beneficia a las empresas, a los consumidores y los ciudadanos.

\section{3 ¿CÓMO PODRÍA LA COOPERACIÓN INTERNACIONAL RESPALDAR LOS PDP EN EL POST CONFLICTO? - LECCIONES APRENDIDAS DESDE LOS LABORATORIOS DE PAZ}

La cooperación internacional, especialmente la europea, tienen un trayecto común con los PDP. La UE jugó un rol importante y confirió aportes fundamentales a los PDP, mediante la consecución de tres Laboratorios de Paz, que abarcan siete regiones del país ${ }^{2}$. Esta iniciativa es el fruto de un "matrimonio" y de la convergencia de intereses y perspectivas políticas en términos de construcción de paz entre los PDP y la UE. Pero, a pesar de que los PDP se figuren como el eje a partir del cual se ha estructurado y desarrollado los Laboratorios de Paz, la UE ha influenciado y transformado las iniciativas en varios dominios.

La posible apertura de una nueva fase de respaldo de la cooperación internacional a los PDP, en un escenario de post conflicto, levanta cuestiones sobre de qué forma y en qué moldes podría esta nueva etapa concretizarse.

Para proyectar y preparar este futuro podemos mirar hacia el pasado. Diez años de colaboración y trabajo conjunto de la cooperación internacional con los PDP permiten tener la distancia analítica para identificar lecciones aprendidas en este proceso, y rectificar y mejorar algunos aspectos en un futuro escenario de post conflicto.

\section{- La importancia del respaldo de la cooperación europea a los PDP:}

Uno de los aportes más importantes de la cooperación internacional a los PDP es que su consolidación y expansión se hizo mediante el apoyo de la cooperación europea, por la vía

2. El primer Laboratorio de Paz se conformó en el Magdalena Medio, el segundo Laboratorio en las regiones del Oriente Antioqueño, Norte Santander, Macizo Colombiano y Alto Patía, y el tercer Laboratorio de Paz en el Meta y los Montes de María. 
de la multiplicación de recursos, con la inyección financiera de la UE. Esto ha contribuido a consolidar y expandir los esfuerzos y proyectos de los PDP y construir una base social más fuerte, permitiendo convocar más organizaciones y alcanzar un número mayor de municipios y personas $^{3}$.

Por lo demás, la presencia de la UE y de actores internacionales ha conferido un cierto blindaje a los procesos sociales de base de los PDP frente a los actores armados, funcionando como una especie escudo y de paraguas políticos.

En esta medida, el respaldo de la cooperación internacional a los PDP en un escenario de postconflicto sigue siendo apremiante.

\section{- La autonomía de la sociedad civil local en el primer Laboratorio de Paz:}

Otro elemento a rescatar de la relación entre la cooperación europea y los PDP fue la autonomía que se confirió a la sociedad civil local, específicamente al PDPMM, en los procesos del primer Laboratorio de Paz. Este se estructuró sobre la base de la experiencia social, el equipo, los principios y la filosofía de paz del PDPMM. La UE nunca ha sido el arquitecto ni el protagonista principal de la iniciativa. El papel de la UE, y especialmente de la Comisión Europea, ha sido de un guía, socio, garantía y verificador, pero no el de una imposición de términos sobre el territorio (Mojica, 2007). El proceso ha sido hecho esencialmente de abajo hacia arriba, manteniendo el Programa un elevado grado de autonomía y control del proceso y la CDPMM el liderazgo y ejecución de la iniciativa (Bayona, 2007), lo que fue uno de los factores de su éxito y sostenibilidad en el terreno.

Esta autonomía de los actores locales de la sociedad civil se perdió, en cierta medida, en la concepción y estructuración del segundo y tercer Laboratorios de Paz, en los cuales hubo una mayor imposición de dinámicas verticales por parte de la UE y del Estado colombiano, que distorsionaron relativamente los procesos sociales para la paz.

Sería importante que, en articulaciones futuras entre la cooperación internacional y los PDP, se volviera a tener en cuenta la centralidad de la sociedad civil en los procesos regionales de construcción de paz, y se rescatara la dinámica del primer Laboratorio de Paz, lo que implica respectar la autonomía política y social de los PDP.

3. Como señala Libardo Valderrama (2007), ex director de la Corporación de Desarrollo y Paz del Magdalena Medio, "el Laboratorio de Paz fue una oportunidad para un impulso. Los recursos iníciales que teníamos eran muy diminutos. Solamente podíamos llegar a un número muy limitado de pobladores y pobladoras. El Laboratorio permitió que llegásemos a un mayor número de personas y que más gente participara en los procesos del Programa." 


\section{- La introducción de dinámicas verticales: las contradicciones entre procedimientos técnicos y realidad social}

La participación de la UE trajo cambios importantes y substanciales a la estructura y metodología de los PDP e introdujo diversas dinámicas verticales a los procesos. Una de las más importantes se sitúa en el plano técnico-administrativo. No obstante, se ha vuelto un importantísimo tema político y social, con efectos negativos en el terreno.

Esta dinámica se relaciona con la conversión de procesos sociales de construcción de paz en proyectos de cooperación al desarrollo, sometidos a los procedimientos técnicos y administrativos establecidos por la Comisión Europea. Particularmente la convocatoria pública exigida por Bruselas, como metodología de selección y financiación de proyectos, ha producido efectos secundarios perversos y fuertes externalidades negativas. A primera vista, es un mecanismo técnico destinado a prevenir la corrupción y establecer transparencia en el proceso. Sin embargo, este elemento se ha transformado en uno de los nudos gordianos de los Laboratorios. En cierta medida, ha contribuido a obstaculizar que los sectores sociales más excluidos de la población participen en los Laboratorios, ya que no tienen la capacidad técnica para formular proyectos, cumplir los requisitos formales exigidos por la UE y tratar con la pesada burocracia europea. El efecto ha sido la participación en los procesos de los Laboratorios de organizaciones ejecutoras de proyectos con alguna dimensión y capacidad instalada, muchas de ellas con experiencia previa en la ejecución de recursos de la cooperación internacional ${ }^{4}$ (Barreto Henriques, 2010).

En la medida en que esta iniciativa de paz pretende ser un mecanismo de inclusión, este factor podría representar que la "cura" sea, de cierta forma, tan mala o peor que la "enfermedad", al convertirse en un mecanismo adicional de exclusión en las regiones y entre las organizaciones sociales (Barreto Henriques, 2009: 576-577).

En el Magdalena Medio este factor ha tenido un efecto negativo adicional y más acentuado, en la medida en que distorsionó y rompió con el proceso y metodología de participación desarrollados por el PDPMM hasta el momento, basado en los "núcleos de pobladores". Estos constituyen espacios populares de participación y ejercicios de planeación participativa, en los cuales organizaciones locales y pobladores de un municipio formulan diagnósticos regionales y

4. Como señala Tito Arbey Pito (2008), del CRIC, "el Laboratorio de Paz le permitió sólo a los grandes, a los que tenían experiencia, poder ejecutar un proyecto". Fanny Medina (2008) cuenta al respecto que "hubo organizaciones que pagaron millones de pesos por la elaboración de los proyectos." En alusión a otro caso sintomático y representativo de esta situación, cuando se le preguntó a un coordinador de un proyecto productivo del Laboratorio del Macizo Colombiano si había tenido dificultades en la formulación de su proyecto en el marco de la convocatoria, su reacción fue: "No. Yo llevo 14 años formulando proyectos. Así, de cierta forma, los Laboratorios de Paz se basan en una "élite" local de movilización y trabajo sociales.

PREPARAR EL POST-CONFLICTO EN COLOMBIA DESDE LOS PROGRAMAS DE DESARROLLO Y PAZ: RETOS Y LECCIONES APRENDIDAS PARA LA COOPERACIÓN INTERNACIONAL Y LAS EMPRESAS 
establecen propuestas de paz y desarrollo, expresas en las propuestas municipales o subregionales (Katz, 2004: 32). El cambio de una metodología eminentemente participativa a una metodología técnica y burocrática produjo muchísimos problemas al proceso social en el Magdalena Medio. Como señala Carlos Moreno (2008: 104), implicó la substitución de una idea y dinámica de democracia deliberativa, incorporada en la metodología de los núcleos de pobladores, por el concepto de democracia competitiva de la UE, explanado en la convocatoria pública.

Los procedimientos técnicos y administrativos requeridos por la Comisión Europea son percibidos en las regiones, por las organizaciones de base, como supremamente pesadas, lentas, inflexibles y altamente burocráticas. Efectivamente, la UE posee una normatividad compleja y 'estandarizada' que difícilmente se adapta a la realidad de algunas regiones colombianas, caracterizadas por su informalidad, fragmentación, baja institucionalidad y pobreza. Como refiere el Padre Eliecer Soto (2007), de la Pastoral Social de Barrancabermeja,

“a veces los proyectos sufren en su ejecución por qué no hay como legalizar o soportar un gasto cumpliendo con todas las normas. No porque no se quiera, pero porque, en una vereda como San Pablo, en Bolívar, por ejemplo, hay gente que no tiene un registro tributario; hay gente que nunca ha salido del monte" ${ }^{\prime 5}$.

Como es visible en este relato, en gran medida, la normatividad de la Comunidad Europea ha chocado con la realidad social colombiana y con la cultura política de las regiones (Barreto Henriques, 2010). Generó una lógica economicista, incoherente con las realidades locales y con los mismos objetivos pretendidos de la iniciativa de paz. La cooperación europea no tuvo en consideración el estado del tejido social e institucional de las regiones donde se implantó. Se concentró en el acompañamiento econométrico de los proyectos y en el cumplimiento de los estándares de los manuales de cooperación europeos, más que en la comprensión de las dinámicas regionales (Moreno, 2008: 102), hecho que imprimió varias dificultades a los procesos y proyectos. Quedó patente así un choque entre lenguajes diferentes y mundos distintos, con evidentes dificultades en dialogar y conciliar 6 .

5. Pero esta realidad se aplica igualmente a las demás regiones del país en donde se han establecido Laboratorios de Paz. Como cuenta Fanny Medina (2008), de la Fundación Sol de Invierno, de Nariño, "En la parte técnica, el marco lógico no es tan lógico y la guía práctica no es práctica. Está llena de minucias. Lo ponen a hablar en euros, en una lógica súper minuciosa y súper medible. Una realidad tan compleja, como la Latinoamericana, no es tan medible. Eso está hecho para un mundo donde todo funciona como un relojito, eso es milimétrico y el mundo latinoamericano no está estructurado ni engranado de esa forma. Si fuera así, no necesitaríamos de un Laboratorio, ni de apoyos internacionales. Por ejemplo, íbamos a presentar un taller, pero cuando llegamos nos dimos cuenta que el 90\% eran analfabetos o descontinuados. Tienes que ir y hacerte un juego para enseñar al otro, te tienes que volver totalmente elástico, y el Laboratorio te dice ¿Cuántos libros escribió, cuántos folletos entregó?"

6. Esta realidad fue reconocida incluso por un funcionario de la Comisión Europea, que afirmó: "yo también alguna vez pensé: pero aquí estamos completamente equivocados se queremos aplicar estas reglas. Pero no teníamos alternativas y todavía no las tenemos". 
Asimismo, los procedimientos y requerimientos técnicos de los Laboratorios de Paz han tenido otro efecto secundario negativo de gran relevancia. Significaron una sobrecarga tremenda sobre los equipos de trabajo de la CDPMM, y de las Entidades Coordinadoras Regionales de los Laboratorios, que sobrepasó su capacidad y personal y, en gran medida, los transformó en gestores y supervisores de proyectos, más que actores políticos y sociales en las regiones (Gómez, 2008).

Estos son factores de enorme peso político y un condicionante esencial para la potencial construcción de paz y resolución pacífica del conflicto armado. Como Libardo Valderrama (2007), ex director de la Corporacion de Desarrollo y Paz del Magdalena Medio (CDPMM), afirma: "el Laboratorio de Paz no pretende ser una empresa de proyectos. Los proyectos son un medio para construir la paz y el desarrollo sostenible". Igualmente, el padre Francisco De Roux (2001), principal ideólogo del PDPMM, ha afirmado que "un laboratorio de paz no es una billetera para financiar proyectos de desarrollo. Sino que es un proceso social, económico y político: busca construir colectivamente una nueva sociedad".

Esta finalidad se enfrenta además a que los timings de los proveedores de fondos de la cooperación internacional tienden a ser de corto plazo. De hecho, el horizonte temporal de la financiación europea a los Laboratorios de Paz es limitado (entre 4 y 8 años). Aunque los Laboratorios se enmarquen, en su concepción y finalidad, en una lógica estructural y de largo plazo que busca incidir sobre las causas profundas del conflicto, su horizonte de pocos años claramente no configura ni permite transformaciones estructurales y choca con las necesidades necesariamente de largo plazo de la construcción de la paz positiva y del desarrollo. Hay un conjunto de micro procesos económicos que no aseguraron sostenibilidad, por el cortísimo tiempo en que se ejecutaron (generalmente entre seis meses y dos años), traduciéndose en iniciativas de valor, pero dispersas y volátiles (Barme y Bouchier, 2008: 88). Como refiere Fanny Medina (2008), "los proyectos de desarrollo pasan a ser proyectos asistencialistas y eso vicia a la comunidad, la daña."

Esta situación configura un riesgo que se estructuren proyectos, más que procesos y que, de esta forma, poco distinga a los Laboratorios de Paz y sus proyectos de una iniciativa de la cooperación internacional "convencional" y "común y corriente" (Barreto Henriques, 2009: 577).

Se configura por lo tanto una situación que encuadra perfectamente en el debate introducido por Mary B. Anderson (1999), en lo cual se refiere a los riesgos y potenciales efectos dañinos de la cooperación y ayuda al desarrollo en escenarios de conflicto. La cooperación europea claramente produjo algunas externalidades negativas en las regiones en donde aportó recursos por vía de los Laboratorios, razón por la cual debe tener en cuenta estos elementos en futuros escenarios de cooperación al desarrollo con los PDP. 


\section{- Un papel político modesto de la UE}

Respecto al rol y participación de la UE en el interior de los Laboratorios de Paz, se manifiesta una tendencia divergente en el plano político. En cierta medida, mientras, a nivel técnico, ha habido "Europa de más", a nivel político, ha habido "Europa de menos". Como señala Calos Moreno (2008: 109), hay un desequilibrio entre los aspectos técnico-administrativos y políticos de los Laboratorios que se ha manifestado en una ausencia de la UE en el terreno y una falta de compromiso político con los Laboratorios. La UE ha ejercido un papel más de "payer" que de "player", es decir, de financiador, y menos de actor político. Esto es notorio esencialmente en dos aspectos.

En primer lugar, se evidencia una cierta ausencia y distancia de la UE, protagonizado por la Delegación de la Comisión Europea en Colombia, de los procesos y vida cotidiana de los Laboratorios. Los funcionarios de la UE tienen una presencia muy reducida (o nula) en el terreno ${ }^{7}$. Su acompañamiento de la construcción de la paz en las veredas y de los proyectos se hace fundamentalmente desde las oficinas de Bogotá. Las visitas de la Delegación a las regiones son breves, y los equipos en las sedes regionales de los Laboratorios son casi exclusivamente colombianos.

Como señala Fernando Valencia (2008), director del Observatorio de Paz y Reconciliación del Oriente Antioqueño, "la UE ha aceptado que fuera el gobierno nacional el que direccionara políticamente el proyecto Laboratorio de Paz. [...] Y se ha alejado del acompañamiento político del territorio." De hecho, la UE ha preferido no comprometer sus buenas relaciones diplomáticas con Bogotá, más que respaldar políticamente la sociedad civil de base. Constituye un ejemplos de esta tendencia la relativa inercia política y falta de reacción pública por parte de la UE frente a casos de violencia o amenazas por parte de grupos paramilitares sobre líderes comunitarios participantes de los Laboratorios, y sobre las violaciones de derechos humanos en las regiones (Bayona, 2007).

Esta situación causa efectivamente alguna desilusión en las organizaciones de los Laboratorios, que reclaman un apoyo político más fuerte por parte de la UE. De hecho, en el contexto

7. Un caso que ilustra esta ausencia de la UE del terreno fue la salida del consejero de cooperación de la Comisión Europea en Colombia, el italiano Nicola Bertolini. Este había sido el principal protagonista e interlocutor europeo en el proceso de los Laboratorios y una personalidad muy comprometida con la iniciativa. Sin embargo, entró en conflicto con el gobierno central y el Gobernador del Departamento del Cauca, Juan José Chaux, volviéndose persona non grata en Colombia, lo que motivó su alejamiento forzado del país. Su salida retiró algún liderazgo y clarividencia políticos a la UE en Colombia y contribuyó a la presencia reducida de la UE en el terreno. Este es de hecho un episodio bien sintomático y representativo del bajo perfil político de la UE en el marco de los Laboratorios y de la resolución pacífica del conflicto armado en Colombia. Han habido evidentes bloqueos y tensiones políticos en la participación de la UE en Colombia, que limitan la acción política de la UE en la resolución del conflicto. 
de inseguridad y amenazas de grupos armados en que se inscriben los Laboratorios, y especialmente el Laboratorio del Magdalena Medio, algunas personas y organizaciones de base solicitan una posición y un compromiso político europeo más sólido, un respaldo político más firme e incondicional con esta iniciativa de paz y una presión sobre el gobierno colombiano que pudiese traer más seguridad al proceso. Como afirma un ex miembro del Laboratorio de Paz del Cauca/Nariño, "la Unión Europea no debe estar más como un donante, sino como un ente internacional que vela por los derechos de un pueblo en crisis; [...], en este sentido debe ser un cooperante político; acá la hemos visto sólo como un donante".

Este es un elemento fundamental que toca tener en consideración: la cooperación internacional debe ser económica y financiera, pero también política.

\section{CONCLUSIONES}

Un potencial escenario de post conflicto en Colombia encierra profundos desafíos políticos, sociales y económicos al país. A la firma de un acuerdo de paz se seguirán largos años de un complicado y exigente proceso de construcción de una paz que se pretende que sea duradera y sostenible y que implica la participación de amplios sectores de la sociedad colombiana.

En este artículo se pretendió subrayar cómo tres actores en particular - los PDP, las empresas y la cooperación internacional, pueden desempeñar un rol fundamental en un contexto de post conflicto en Colombia.

Los PDP constituyen modelos consolidados de construcción de paz desde la sociedad civil y las regiones, con una experiencia acumulada de casi dos décadas de trabajo junto a las comunidades más carenciadas. Su enfoque multidimensional para la paz ha producido resultados e impactos considerables en el territorio en un contexto de violencia armada. La apertura de un escenario de post conflicto incrementaría sus posibilidades de trabajo para la paz y su importancia en cuanto actores regionales de construcción de paz.

Podrían configurar instrumentos válidos para que los acuerdos "bajen" hasta las regiones y a las comunidades que vivenciaron décadas de violencia. Es fundamental que la construcción de la paz en el post conflicto encierre un matiz y un enfoque regional, pues Colombia es regionalmente diferenciada, tal como sus instituciones y su violencia armada. En este sentido, el potencial de los PDP es inmenso, en cuanto procesos regionales de construcción de paz. Abarcan elementos que, si fueran desarrollados e incrementados en una escala más amplia, podrían construir los cimentos para la paz durable y positiva en el país.

La cooperación internacional y las empresas podrían figurar como aliados en este proceso. Construir la paz en Colombia tendrá costos, a nivel político, social, pero también financiero. 
Estos actores tienen la capacidad para asumirlos, direccionando recursos para la generación de una economía de paz y la construcción de un país y una sociedad en paz, por vía del respaldo político y financiero a las iniciativas de los PDP. Esto sería esencial a fin de garantizar su buen funcionamiento y sostenibilidad, así como potenciar su rol en una etapa de post conflicto.

\section{REFERENCIAS}

- Anderson, M. (1999). Do no harm: How aid can support peace - or war, Londres: Lynne Rienner.

- Bouchier, J., Barme, C. (2008). Informe Final Evaluación Intermedia del Programa Segundo Laboratorio de Paz, Bogotá, 29 de octubre.

- Barreto Henriques, M. (2009). "El Laboratorio de Paz del Cauca/Nariño: una salida indígena para la paz en Colombia?" in Restrepo, Jorge; Aponte, David (eds.) Guerra y violencia en Colombia: herramientas e interpretaciones, Bogotá: Editorial Javeriana.

- Barreto Henriques, M. (2010). "El rol de la UE en la resolución del conflicto en Colombia: un análisis desde el terreno", Revista Aldea Mundo, Año 15, № 29, enero-junio.

- Barreto Henriques, M. (2012). “"Laboratorios de Paz" en territorios de violencia(s): abriendo caminos para la paz positiva en Colombia?", tesis de doctorado presentada en la Universidad de Coimbra.

- Burton, J. (1990). Conflict Resolution and Provention, New York: St. Martin's Press.

- De Roux, F. (2001). "Un Laboratorio de Paz en el Magdalena Medio", Barrancabermeja: PDPMM, 7 de mayo.

- Galtung, J. (1996). Peace by peaceful means: Peace and Conflict, Development and Civilization, London: Sage Publications.

- Granada, S.; Restrepo, J.; Vargas, A. (2009). "El agotamiento de la política de seguridad: evolución y transformaciones recientes en el conflicto armado colombiano", in Restrepo, J.; Aponte, D. (eds.). Guerra y violencia en Colombia: herramientas e interpretaciones, Bogotá: Editorial Javeriana.

- Katz, M. (2004). "Experiencia regional de paz: El Programa de Desarrollo y Paz del Magdalena Medio", Controversia, no 181, CINEP, febrero, Bogotá. 
- Lederach, J. P. (1997). Building Peace. Sustainable Reconciliation in Divided Societies, Washington DC: United States Institute of Peace Press.

- McDonald, G. (1997). "Peacebuilding from below. Alternative perspectives on Colombia's peace process", London: Catholic Institute for International Relations.

- McDonald, Geraldine (1998), Alternative Perspectives on Building Peace in Colombia and El Salvador: An appraisal of the peace processes with special reference to peacebuilding from 'below', PhD Thesis, Department of Peace Studies, University of Bradford.

- Moreno, C. (2008). "Laboratorios de Paz: una política de creación", Análisis Político, 65, septiembre/diciembre.

- Mouly, Cécile (2011) "Peace Constituencies in Peacebuilding: The Mesas de Concertación in Guatemala", in Pugh, Michael; Cooper, Neil; Turner, Mandy (eds.) Whose Peace? Critical Perspectives on the Political Economy of Peacebuilding, Houndmills: Palgrave Macmillan.

- Richmond, O. (2008). Peace in Internacional Relations, Londres: Routledge.

- Saavedra, M. R., Ojeda, L. (2006). Trabajo en Red: Imaginarios conceptuales de paz, desarrollo y región en los programas de la Red Prodepaz, Documentos Ocasionales n. 74, Bogotá: CINEP.

- Sousa Santos, B. (2003). Democratizar a Democracia: Os Caminhos da Democracia Participativa, Porto: Edições Afrontamento.

\section{ENTREVISTAS}

- Bayona, M. (2007). Ex subdirector técnico de la CDPMM. Entrevista por teléfono BogotáBucaramanga, 25 de agosto.

- Gómez, A. (2008). Antropólogo, ex Coordinador Técnico Regional del Laboratorio de Paz del Macizo Colombiano. Popayán: 14 de febrero de 2008.

- Medina, F. (2008). Directora de la Fundación Sol de Invierno. Pasto: 15 de octubre.

- Mojica, A.M. (2007). Funcionaria de la Delegación de la Comisión Europea en Colombia, task manager del Laboratorio de Paz I. Bogotá: 6 de septiembre.

- Pito, T. (2008). Ex asesor económico del CRIC, funcionario de la ECR del Laboratorio de Paz del Macizo. Popayán: 20 de octubre. 
- Soto, E. (2007). Sacerdote de la Diócesis de Barrancabermeja, miembro del PDPMM. Barrancabermeja: 23 de mayo.

- Valderrama, L. (2007). Padre jesuita, ex director de la CDPMM. Barrancabermeja: 24 de mayo.

- Valencia, F. (2008). Director del Observatorio de Paz y Reconciliación del Oriente antioqueño. Rionegro: 2 de diciembre.

- Vincenti, F. (2008). Ex coordinador de la línea 1 de la Asistencia Técnica Internacional al Segundo Laboratorio de Paz. Bogotá: 26 de febrero. 